\title{
LE QUEBEC ET LA COUR SUPREME
}

\author{
PIERRE PATENAUDE*
}

The fact that the Supreme Court Judges are named by the Federal Executive is contrary to the basic rule of federalism which imposes an impartial arbiter. When fudges who have to decide on political and constitutional issues are chosen unilaterally by one of the litigants, the presumption of impartiality is lessened. Perhaps the recent centralist decisions of the Supreme Court of Canada would have been different if the provinces would have had the possibility of nominating a certain number of fudges. For Quebec, the National State of French Canadians, the defense of its furisdiction is a vital question for the survival of its culturel For this province, a centralist Supreme Court becomes a major danger in the evolution of the Canadian Constitution.

P.P.

Dans une situation de minorisation rapide et, semble-t-il irréversible, le peuple québecois nécessite à sa survie un pouvoir de gestion exclusif de tout domaine à caractère culturel. Selon Statistique Canada, le Québec ne compterait que 22 à $24 \%$ de la population canadienne en l'an 2,000, lui permettant d'avoir une soixantaine de députés aux communes fédérales. Jointe à l'assimilation galopante des francophones des autres provinces, cette faiblesse numérique amènera nécessairement une perte d'influence au niveau fédéral.

Or, alors que les francophones comptaient, depuis 1867, pour environ le tiers de la population canadienne, ils ont vu le gouvernement canadien laisser se réaliser leur assimilation complète dans plusieurs provinces par l'abolition de leur système scolaire et un harcèlement continuell Ce n'est que depuis quelques années que la majorité candienne découvre le bienfondé d'une politique protectrice des minorités, alors que la jeunesse française de ces provinces anglo-saxonnes ne parle souvent plus que quelques mots de la langue de Molière!

Evidemment, le peuple québecois ne peut, dans un tel contexte, confier les outils de sa survivance culturelle à un Parlement majoritairement de langue et de culture différentes. Ce peuple minoritaire n'acceptera de faire partie d'une Confédération économique qu'à la condition de conserver une juridiction exclusive sur les domaines essentiels à sa survie.

Pour sa protection contre un pouvoir central sans cesse envahissant, le Québec pouvait compter jusqu'à récemment sur la possibilité d'en appeler d un arbitre impartial: le Comité judiciaire du Conseil privé de Londres. Celui-ci dut, d'ailleurs, mettre constamment un frien aux velléités expansionnistes du pouvoir fédéral, pour donner à la Constitution canadienne une certaine apparence de fédéralisme. On sait la suite de l'histoire: les décisions provincialistes de 1937 mirent le feu aux poudres. On assista alors à un tollé chez les anglocanadiens, dont les critiques virulentes amenérent l'abolition des appels à Londres. Les arguments des tenants de l'abolition des appels au Conseil privé furent d'ailleurs repris par l'actuel juge en chef Laskin dans ses écrits antérieurs à sa nomination. ${ }^{1}$

- Avocat, Professeur agrégé. Président de la section de droit constitutionnel de l'Association des professeurs de droit du Canada.

1 Laskin, Canadian Constitutional Law: Carswell, (1960) p. 28-39 at 38: "There is strong opinion that what is required in Canada at the present time is a re-allocation of powers, something which only amendment can achieve. If that is so, it points up some failure at least on the part of the courts - and especially the Privy Council which was in command for almost 85 years following Confederation - to discharge the task of keeping the B.N.A. Act in tune with contemporary needs."

See also Laskin, The Supreme Court of Canada: A final Court of and for Canadians, (1951) 29 Can. Bar Rev. 1038. 
La Cour suprême du Canada devenait l'arbitre final des conflits constitutionnels en 1949. Mais quel arbitrel Tribunal créé par une loi du pouvoir central, formé de juges choisis par ce même pouvoir, siégeant dans la capitale fédérale avec obligation pour ses membres d'y résider! L'apparence d'impartialité n'est pas très forte, d'ailleurs plusieurs constitutionnalistes étrangers en conviennent. ${ }^{2}$

Les effets d'une telle décision ne se firent d'ailleurs pas attendre longtemps: dès 1950, ce Tribunal réaffirmait le pouvoir du parlement fédéral de légiférer sur toute matière de compétence provinciale, lors d'urgence nationale, allouant pour la premiére fois, à ce pouvoir politique, le droit de définir lui-même cet état d'urgence et de le prolonger aussi longtemps qu'il le jugerait nécessaire. ${ }^{3}$ On créait un outil supplémentaire d'assujettissement de la minorité québécoise. Le droit octroyé au pouvoir central de définir unilatéralement ce qu'est un état d'urgence équivalait alors à confier un pouvoir tout à fait arbitraire à la majorité et une possibilité d'ingérence supplémentaire dans le domaine de juridiction des provinces.

Ainsi, s'agirait-il d'une urgence nationale si le peuple québecois utilisant son droit inaliénable et reconnu par les Nations-Unies à l'auto-détermination," décidait de se séparer du reste du Canada? Serait-ce un état d'urgence nationale si un parti politique prônant une telle sécession était démocratiquement élu?

Oserait-on se servir de cet outil juridique pour contrôler l'exploitation des richesses naturelles lors de crise énergétique?5 Autant de questions que peut se poser la minorité québecoise.

En 1951, on établissait le principe que les Etats provinciaux ne pouvaient nuire aux droits de citoyens canadiens de circuler à travers le pays. ${ }^{\circledR}$ Cette décision pourrait-elle bloquer une politique québecoise visant à empêcher une assimilation rapide par l'afflux continuel de citoyens d'autres provinces? Pourtant tout peuple qui se respecte doit légiférer pour protéger, jusqu'à un certain degré, son homogénéité culturelle.

La Cour suprême, dans un même esprit de centralisation, avait ravivé dès 1952 la théorie des dimensions nationales; ${ }^{7}$ en 1966, elle extensionna cette théorie jusqu'à l'absurde: considérant que l'existence de parcs dans le capitale nationale était nécessaire da la vie même du Canada et était une question de portée nationale, elle reconnut à la Commission de la Capitale nationale le pouvoir d'exproprier certaines propriétés privées sur le territoire ontarien. ${ }^{8}$ Inutile d'insister sur le fait qu'une telle décision permettait à cette Commission de faire de même au Québec. On donnait à une autorité dépendante d'un pouvoir étranger, le droit de gruger

2 E.g., Wheare, Federal Government, 3rd ed., (1953-1961) at 68: "The Case of Canada, where the appointment of all judges is in the hands of the general government, is an example of a system which contradicts the federal principle."

See also, Durand, "L'Etat fédéral en droit positif" dans Institut d'études juridiques de Nice: Le Fédéralisme, (1956) at 188.

${ }^{3}$ Reference as to Validity of the Wartime Leasehold Regulations, [1950] S.C.R. 124.

4 Pacte international relatif aux droits civils et politiques, art.l: "Tous les peuples ont droit de disposer d'eux-mêmes. En vertu de ce droit, ils déterminent librement leur statut politique et assurent librement leur développement économique, social et culturel."

- On sait d'ailleurs que le pouvoir fédéral utilisa récemment l'excuse de l'urgence nationale pour légiférer sur les richesses naturelles provinciales. (Bill C-236, 21-22 Eliz. II, 1973.)

6 Winner v. S.M.T. (Eastern) Ltd. \& A.G. of Canada [1951] S.C.R. 887.

7 Johanneson v. Rural Municipality of West St. Paul [1952] I S.C.R. 292.

8 Munroe v. National Capital Commission (1966) 57 D.L.R. 753, [1966] S.C.R. 663. 
le territoire nationale des québécois. ${ }^{\circ}$ Un élargissement excessif de cette théorie des dimensions nationales amène de plus l'affaiblissement du principe fondamental d'un véritable fédéralisme, celui d'une scission de souveraineté, en permettant à l'Etat central d'envahir le domaine de juridiction des provinces dès que semble exister un intérêt tant soit peu extra-provincial.

Plusieurs autres jugements vinrent étayer cette ligne de conduite axée sur une augmentation des pouvoirs fédéraux: citons, entre autres, les affaires Murphy v. C.P.R. ${ }^{10}$ In re Offshore Mineral Rights of British Columbia, ${ }^{11}$ et Caloil v. P.G. du Canada ${ }^{12}$ dans lesquels, soit par application de la théorie de l'ancillarité ou par extension exagérée de la juridiction fédérale en matière de relations internationales, on lui augmenta sa juridiction au détriment des provinces. Comme la question du partage des compétences fut étudiée dans un autre atelier, nous ne nous y arrêterons pas.

Plusieurs se sont surpris de cette tendance nettement centralisatrice de la Cour suprême. Il n'y a pourtant rien d'étonnant dans ses décisions. En effet, il est inutile de spécifier que l'adresse politique des éminents juristes à être nommés à ce haut Tribunal est scrutée par le gouvernement fédéral. Pour ne donner qu'un seul exemple, mentionnons que les juges Fournier, Sedgwick, Davies, Fitzpatrick, Brodeur, Mills et Abbott avaient été ministres du gouvernement fédéral avant leur nomination; seuls les juges Lamont et Estey avaient été ministres dans des gouvernements provinciaux. ${ }^{13}$ Il serait pour le moins surprenant que d'honnêtes hommes luttent pour un idéal alors qu'ils sont en politique et appliquent, lors d'incertitude, une philosophie toute autre dès qu'ils montent sur le ban.

En outre, tous doivent demeurer à Ottawa pendant leur séjour à la Cour. Or, on sait l'influence que peut avoir un milieu social sur les hommes: des amis ou voisins viendront, involontairement, soutenir dans une réunion amicale leur point de vue sur tout sujet politique; les journaux, la radio, la télévision véhiculeront leur option politique. Or la capitale fédérale est loin d'être le refuge des décentralisateurs!

Les juges de la Cour suprême semblent donc souvent placés aussi loin de la pensée québécoise que ne l'étaient les juges du Comité judiciare du Conseil privé de la canadienne-anglaise!

Comprenons bien qu'il ne s'agit pas ici de mettre en doute l'honnêteté et la compétence des juges de cette Cour, mais, de constater que comme tout autre citoyen, leur expérience antérieure et l'influence du milieu peut grandement influencer leurs choix politiques.

Face à une telle situation, comment s'étonner de l'unanime critique de la méthode de nomination à ce Tribunal par les juristes et hommes politiques québécois: mentionnons les premiers ministres Duplessis, ${ }^{14}$ Lesage $^{15}$ et John-

- Brossard, "La région du district fédéral," dans Brossard, Immarigeon, LaForest et Luce Patenaude, Le Territoire québécois (P.U.M., 1970) pp. 273-313.

10 [1958] S.C.R. 626.

11 [1968] 65 D.L.R. 353. See also Brossard, "La Cour suprême, l'Etat fédéral et les Gisements miniers sous-marins," in Le Territoire québécois, supra, n. 9, at 152.

12 [1971] S.C.R. 543.

13 Adams and Cavalluzo, The Supreme Court of Canada, a Biographical Study (1969) 7 Osgoode Hall L.J. 61 at 69.

14 Conférence fédérale-provinciale de 1950; comte-rendu de la conférence fédéraleprovinciale au sujet de la Constitution, Québec, 25-28 septembre 1950, Ottawa, Imprimeur de la Reine, 101.

15 Conférence fédérale-provinciale 1960, Ottawa, Imprimeur de la Reine, 33. 
son, ${ }^{16}$ les juristes Jacques-Yvan Morin, ${ }^{17}$ maintenant chef de l'opposition officielle du Québec, Gérald Beaudoin, ${ }^{18}$ Jacques Brossard, ${ }^{19}$ André Tremblay ${ }^{20}$ et Edward McWhinney. ${ }^{21}$ Le Rapport de la Commission Royale d'enquête sur les problèmes constitutionnels de $1956^{22}$ avait d'ailleurs fortement insisté sur l'importance d'une impartialité totale du Tribunal constitutionnel pour la survie même du peuple québecois. Les Etats généraux du Canada français reprirent d'ailleurs ce thème. ${ }^{23}$

On peut comprendre, de plus, l'opposition du Québec à l'inclusion dans le texte constitutionnel d'une Déclaration canadienne des droits de l'homme. Sa méfiance vis-à-vis du Tribunal qui aurait à appliquer le texte fondamental pousse l'Etat québecois au refus de lui octroyer des outils supplémentaires d'ingérence dans la politique interne du Québec. ${ }^{24}$

Il est intéressant de s'arrêter quelques instants pour noter le peu d'influence que peut avoir un peuple minoritaire sur un gouvernement central contrôlé par l'autre groupe national: depuis au moins vingt-cinq ans, les demandes en faveur d'une réforme de la Cour suprême sont unanimes du côté francophone. Or, rien n'a été fait. D'un autre côté, lorsque les anglophones canadiens exigèrent l'abolition des appels au Comité judiciaire du Conseil privé, ce, à l'encontre des intérêts québecois, ils furent exhaussés après une dizaine d'années de lutte.

Le Québec ne peut accepter que le tribunal constitutionnel soit formé de juristes choisis unilatéralement par le gouvernement central: c'est donner à ce dernier un pouvoir trop grand! N'avons-nous pas vu Roosevelt nommer des juges partisans à la Cour suprême des Etats-Unis dans le but avoué d'y faire accepter son New Deal? Qu'est-ce qui empêcherait un gouvernement central fort de faire de même pour imposer une constitution unitaire au Canada et détruire ainsi la culture d'une minorité souvent encombrante?

Ce danger, pour la minorité québecoise, s'accroît d'ailleurs lorsque la Cour suprême opte pour une interprétation évolutive du texte constitutionnell En effet, tant et aussi longtemps que ce Tribunal se sentait lié au stare decisis des décisions antérieures, il devait respecter la fédéralisation du système politique canadien consacrée par le Comité judiciaire du Conseil privé.

${ }^{16}$ Gouvernement du Québec, Exposé préliminaire, Conférence sur la Confédération de Demain (Toronto, 27-30 novembre 1967), 23.

17 Morin, Le Québec et larbitrage constitutionnel: de Charybde á Scylla (1967) 45 Can. Bar Rev. 608.

18 Beaudoin, La Cour suprême du Canada et le Constitutionnalité des lois, Journées d'études juridiques Jean Dabin, 1973, Bruxelles, 187. Beaudoin, La revision constitutionnelle et le statut de la Cour supréme (1970) 1 Rev. Gen. Dr. 257.

19 Brossard, La Cour suprême et la Constitution, (P.U.M. 1968), 427.

20 Tremblay, Les techniques d'interprétation des Constitutions (1967) 5 Coll. Int. dr. comp. 49 at $50-51$.

21 McWhinney, Federal Supreme Court and Constitutional Review (1967) Can. Bar Rev. 591-93.

22 Commission Tremblay, province de Québec 1956, vol. 2, pp. 101 et 107; 161-163, 168-70; vol. 3(1) pp. 295-301.

23 Les Etats généraux du Canada français, Assises nationales 1967, p. 79.

24 Honorable Daniel Johnson, Conférence fédérale-provinciale 5 au 7 février 1968: "Nous avons déjà indiqué à plusieurs reprises que la composition de la Cour suprême la rendait difficilement acceptable comme cour constitutionnelle de dernier ressort. Une nouvelle constitution devra prévoir ce point important avant que nous puissions consentir à une déclaration constitutionnelle des droits." 
Aujourd'hui, de fortes pressions ${ }^{25}$ sont exercées pour que la Cour suprême, à l'exemple du tribunal américain, adapte la Constitution à l'évolution politique du pays et déroge en conséquence, aux règles établies précédemment. ${ }^{26}$ Fort bien! Mais encore faut-il que ses membres ne perçoivent pas tous l'évolution souhaitée dans un sens unique.

Beaucoup a été écrit sur le sujet, rien n'a été fait en réponse. Ce n'est certes pas en prenant l'avis de la Canadian Bar Association sur le choix des juges qu'on donne voix au chapitre à la minorité nationale. Serait-ce qu'on préférerait que le Québecois cesse ses récriminations et décide de porter les conflits futurs devant les organismes internationaux chargés de régler les conflits entre Etats souverains? La nation québecoise acceptera peut-être de continuer son apport à l'expérience canadienne, mais à la condition d'y jouer à titre de partenaire égal à l'intérieur d'un jeu où les dés ne sont pas pipés d'avancel

Chaque critique de la composition de notre tribunal constitutionnel s'est plu à apporter une solution à ce dilemne. Je ne ferai pas exception à la règle.

Le Canada est un pays composé de dix provinces mais aussi de deux groupes culturels distincts. A ce titre, le Pouvoir qui représente les deux collectivités et les dix provinces devrait refléter cette double réalité. Face à une chambre basse élue proportionnellement à la population, il devrait exister un sénat bi-national dont les membres, nommés en partie par les provinces, seraient en nombre égal des deux groupes linguistiques. On pourrait prévoir trois composantes à ce Sénat: le tiers serait nommé par le Québec, un autre tiers par les autres provinces et, enfin, la dernière partie représenterait les minorités anglophones du Québec et francophones des autres provinces à parts égales et serait choisie par l'exécutif fédéral.

Ce Sénat aurait trois fonctions principales: un droit de véto suspensif sur toute législation fédérale ayant quelque portée sur l'aspect culturel du Canada, la nomination des juges de la Cour Suprême du Canada et un pouvoir d'enquête sur la situation accordée aux minorités canadiennes.

Quant au Tribunal, il serait composé de quinze membres: cinq québecois de formation civiliste et dix canadiens de Common Law. Ainsi, toute cause civile émanant du Québec serait toujours jugée par un ban formé en très grande majorité de membres de formation civile.

Le Sénat bi-national choisirait les juges de la Cour suprême parmi des noms présentés par les autorités politiques canadiennes: trois des juges québecois seraient choisis d'une liste préparée par le gouvernement du Québec, deux de celle dressée par l'Exécutif fédéral. Quant aux juges anglo-saxons, deux seraient pris d'une liste présentée par l'Ontario, deux par l'ensemble des provinces de

25 Weiler, In the Last Resort, (Toronto: Carswell, 1974), p. 246. See also Laskin, Canadian Constitutional Law, supra, n. 1, at 38: "In Canada, it would seem that the gap between what is required and what judicial interpretation has given us is, in many cases, so wide as to support the conclusion that only amendment can bridge it - unless, of course, the Supreme Court of Canada (now the ultimate judicial authority) is bold enough to reverse or deflect the authority of Privy Council decisions. Some erosion of those decisions is already evident but one cannot be categorical about this matter."

${ }^{26}$ Laskin, The Supreme Court of Canada: a Final Court of and for Canadians, (1951) Can. Bar Rev. 1038, at 1069: "The Supreme Court is now free to adopt its own canons of judicial behaviour . . . . . It is worth remembering that for a final court consistency in decisions is merely a convenience and not a necessity. No one expects the Supreme Court to break out in rash reversals of previous holdings, even if it should formally dissociate itself from stare decisis. In my view, such a dissociation, whether formally expressed or not, is imperative if the court is to develop a personality of its own. It has for too long been a captive court." 
l'Ouest, un par les provinces maritimes et cinq d'une liste présentée par le fédéral. Chaque composante de la mosaique canadienne serait ainsi représentée, les deux tendances politiques présentes et une apparence d'impartialité imposerait le respect de ce Tribunal à tous les Canadiens.

Cette solution n'en est cependant qu'une parmi plusieurs et exigerait une revision constitutionnelle. Il serait entre-temps possible de satisfaire partiellement les revendications des décentralisateurs en prévoyant, temporairement, que, tout en gardant la même structure du Tribunal, l'Exécutif fédéral soit tenu d'en choisir la moitié des juges à l'aide d'une liste préparée par les autorités provinciales.

Ce serait déjà témoigner d'une bonne foi dans l'élaboration d'une revision constitutionnelle et prouver aux Québécois que leurs revendications ont quelque chance d'être écoutées!

Les français du Canada y sont partout minoritaires. Cependant les exigences de cette minorité différent selon qu'il s'agit de la minorité québécoise, majoritaire dans son Etat national et maîtresse de ses destinées, ou de celle des autres provinces canadiennes, minorités culturelles. Cette dernière ne peut exiger que la reconnaissance de certains droits limités tels l'enseignement dans sa langue et la pratique de sa religion.

Au contraire, la minorité nationale, contrôlant un Etat, aura le désir de se gouverner elle-même et de progresser dans sa culture.

Déjà en 1867, le compromis canadien lui reconnaissait, en partie, ce fait.

Si l'arbitre constitutionnel bloque le québécois dans ses aspirations fondamentales ou encore s'il donne une trop grande impression de partialité, ce sera peut-être un argument supplémentaire pour terminer l'expérience centenaire d'une union politique qui est devenue un simulacre de fédéralisme. 